\title{
Filter Leak Tester for Semiconductor/LCD Clean Rooms
}

\author{
Shintaro Sakamoto \\ R\&D Center \\ Shinryo Corporation \\ 41, Wadai, Tsukuba, Ibaraki, 300-4247, Japan \\ sakamoto.sh@shinryo.com
}

\author{
Kouetsu Tanaka \\ R\&D Center \\ Shinryo Corporation \\ 41, Wadai, Tsukuba, Ibaraki, 300-4247, Japan \\ tanaka.ko@shinryo.com
}

\begin{abstract}
Previous leak tests for ceiling filters of semiconductor/LCD clean rooms were performed manually and so it was difficult to reduce their time and cost. Therefore, we developed a filter leak tester with an automatic probe scanner and a manual lift mechanism on a truck. One of the meritsof the tester is a mask for isolating air blowing in from outside. The leak test can be easily performed without setting polyethylene sheets to mask each filter. In this paper, we describe the specifications and functions of the filter leak tester. We also present the results of using the tester at the actual construction site of a semiconductor clean room, and evaluate the effects.
\end{abstract}

Keywords: Clean Room, Filter Leak Test, Particle Counter, Scanning

\section{INTRODUCTION}

Many robots and systems were developed in the 1980s and 1990s to automate various works at construction sites. We developed a robot for marking on ceiling boards ${ }^{[1][2]}$ and a robot for measuring outlet air volume ${ }^{[3][4]}$ to automate the construction of air-conditioning systems. These robots move autonomously andhave numerous superior functions, for example, automatically measuring self-position in a room and detecting outlets using CCD camera vision. However, they have never been used at actual construction sites, mainly because the environment is not suitable for robots, for example, due to materials and scaffolding on the floor. Needless to say, it is very important to continue to develop the robots for construction of air-conditioning system. However, we also considered it possible to improve the efficiency by using automatic machinesoperated by humans rather than using the autonomous robots presently available. Therefore, we began developing several machines to reduce the construction costs by decreasing the time and amount of manual labor.

In the construction of semiconductor/LCD clean rooms, ceiling filters should be tested to confirm the absence of leakage. However, performing the test manually is extremely difficult, preventing any reduction in time and cost. Robots for performing filter leak test have been developed since the $1980 \mathrm{~s}^{[5][6]}$, but typically weigh more than $250 \mathrm{~kg}$ due to the required movement functions and power source for conducting a fully automatic test. Such a heavy robot is difficult to carry into and out of a construction site and move between rooms. We developed an automatic filter leak testing machine for human operation and used it at an actual construction site. This machine has an XY scanner with servomotors, a manual lift mechanism and four casters. The operator moves the machine to a point under the filter, raises the XY scanner and conducts the filtertest. One of the merits of the machine is that it is possible to set a mask for isolating air blowing in from outside. Clean rooms that have a low level of cleanliness, even those under construction, can still be tested. In the past, polyethylene sheets had to be set as a mask under the filter, adding to the total cost.

In this paper, we firstexplain the standard for the filter leak test method provided bythe Japan Air Cleaning Association (JACA) and outline the specifications and test method of our machine following the standard. Next, we present the results of using the machine in a large-scale non-unidirectional airflow clean room that has filters with diffusers, and evaluate the effects

\section{METHOD USED FOR FILTER LEAK TEST}

\subsection{JACA Standards}

The method used for the filter leak test in clean rooms is provided in JACA Standard No. 24-1989. This machine was developed following the standard, part of which appears below.

(1) Apparatus for airborne particle count

An apparatus can be selected from among the alternatives shown below:

i) Aerosol photometer with linear or logarithm readout, a nominal sampling flow rate of which is more than $28.3 \times 10^{-3} \mathrm{~m}^{3} / \mathrm{min}(1 \mathrm{CFM})$, sensitivity of more than $1 \mu \mathrm{g} / \mathrm{m}^{3}$ at a test aerosol that the diameter is $0.3 \mu \mathrm{m}$, and a measurement range of up to $10^{5}$

ii) A light scattering particle counter with a nominal sampling flow rate of $0.01 \mathrm{~m}^{3} / \mathrm{min}$ and a sensitivity of more than 0.3 $\mu \mathrm{m}$

iii) A light scattering particle counter with a nominal sampling flow rate of $28.31 / \mathrm{min}$ and a sensitivity of more than $0.5 \mu$ $\mathrm{m}$.

(2) Test method (filter leak scan test)

After the aerosol upstream is controlled to the established concentration, the operator should use the sampling probe for scanning to detect the leakage. The scanning points include the surface of the filter, the seal between the filter and the filter fixing frame, and the joint of said frame. As shown in Figure 1, the probe should be scanned at a distance of less 
than $25 \mathrm{~mm}$ from the filter and the test point at a velocity of less than $50 \mathrm{~mm} / \mathrm{s}$. The probe aperture should enable inlet air velocity to be approximately the same as or somewhat faster than filter exit airflow velocity. Scanning stroke should be calculated so that scanning traces slightly overlap.

(3) Acceptance criteria

i) For an aerosol photometer

When there is a reading of over $10^{-4}$ times the concentration of aerosol upstream or when, with a linear readout photometer, readout exceeds 1 point of the scale, leakage should be judged and reported.

ii) For a light scattering particle counter

a) HEPA filter

When upstream concentration of $3 \times 10^{-4}$ times or higher is counted continuously with particles of $0.3 \mu \mathrm{m}$ in diameter or higher in one place or upstream concentration of $1 \times 10^{-4}$ times or higher is counted continuously with particles of $0.5 \mu$ m ormore in one place, it shall be regarded as leakage, recorded and reported.

b) ULPA filter

The acceptance criteria should be determined in an agreement between the owner and the producer.

\subsection{Test issues}

As shown inFig. 2, this filterleak test has been performed manually up until now. It is an extremely difficult test that requires a high level of skill because the entire surface of the filter must be scanned while maintaining the specified velocity as well as distance from the filter. Furthermore, the scanning and measuring process requires two operators.

In addition, the test had to be done after setting polyethylene sheets on the filters to isolate air blowing in from outside in the case of clean rooms with non-unidirectional airflow or in the case construction was being performed simultaneously due to scheduling. Therefore, the work for setting the sheets increased the time and cost.

\section{SPECIFICATIONS AND TEST PROCEDURE}

3.1 Machine requirements

(1) Test object Ceiling filters in clean room

(2) Probe scanning

i) Possible to scan at a constant velocity of less than $50 \mathrm{~mm} / \mathrm{s}$

ii) Possible to maintain specified distance from the filter

iii) Possible to perform particle count and leakage detection automatically

(3) Manpower

Possible for single operator to perform the filter leak test

\subsection{Machine structure and operation}

A picture of the machine is shown in Fig. 4 and the specifications are listed in Table 1. The machine is composed of the probe scanning mechanism, which scans with the sampling probe in two directions crossing at right angles $(\mathrm{X}, \mathrm{Y})$ using two servomotors, the lift truck that raises

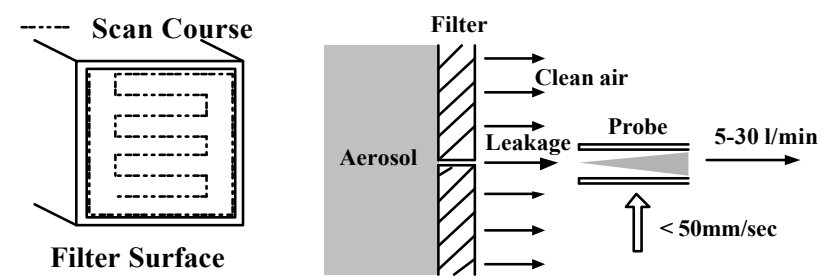

Fig. 1 Scanning method for HEPA filter

(JACA No. 24-1989)

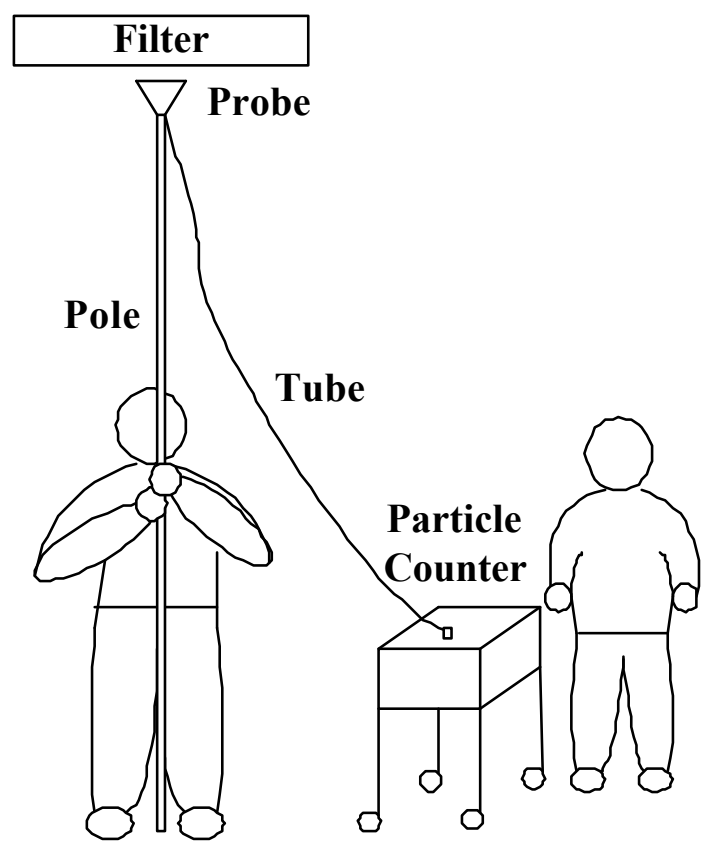

Fig. 2 Conventional filter leak test

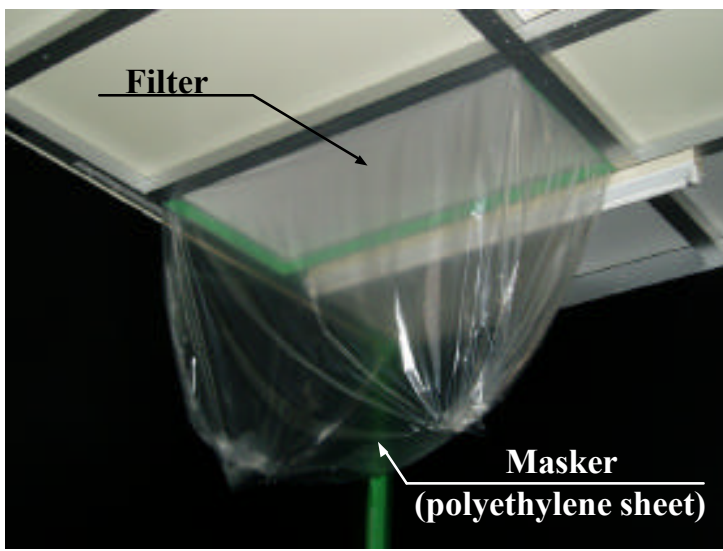

Fig. 3 Example of setting masker

the probe scanning mechanism up to the filter, and the system that controls the motors and the particle counter and judges the status of leakage based on data from the particle counter. In addition, there is a fence to isolate air blowing fromoutside. We refer to this fence as the "Masker".

The probe scanning mechanism scansin the XY direction using two servomotors, timing belts, pulleys, guide rails and ball screws. It has both an automatic and a manual scanning mode. 


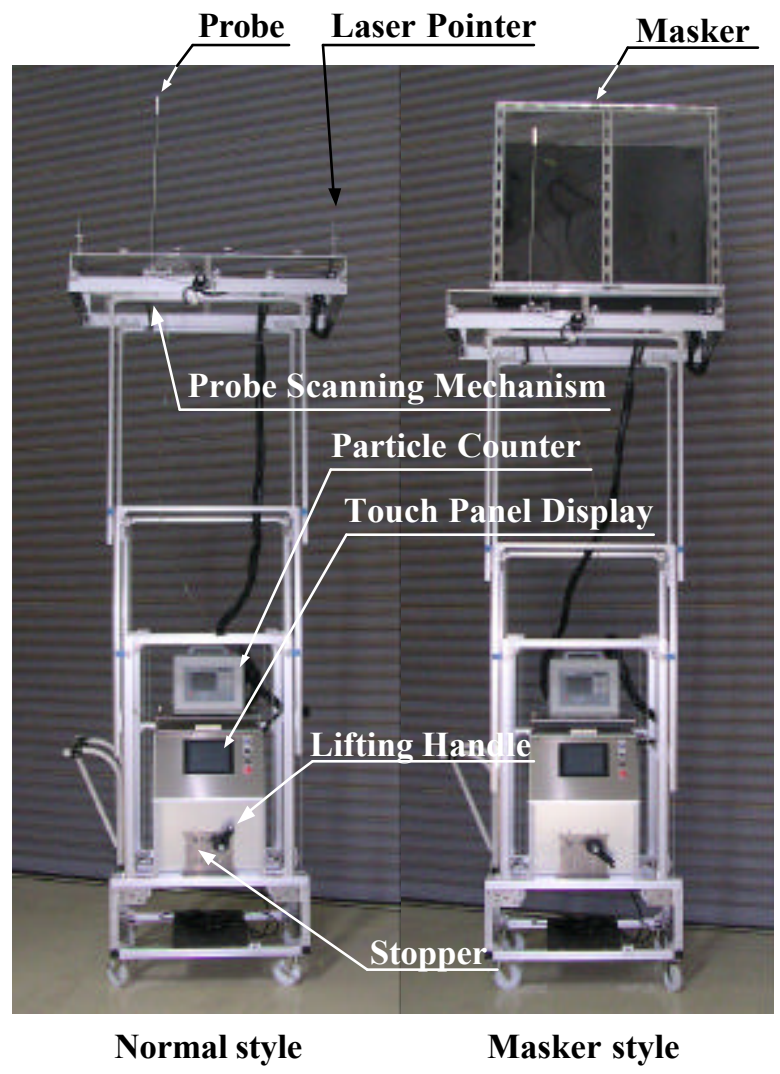

Fig. 4 Filter leak tester

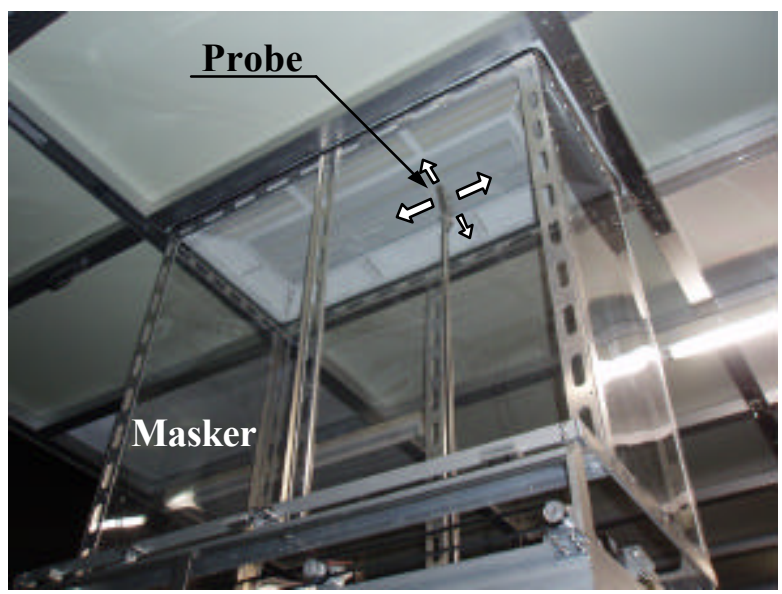

Fig. 5 Aspect of leak test

The former mode scans the entire surface of the filter with the probe at a constant velocity $(50 \mathrm{~mm} / \mathrm{s})$ and detects leakage automatically. The scanning pitch of the probe is less than the probe width for overlapping the traces, and the width of overlapping can be optionally set from 0 to $10 \mathrm{~mm}$. For the velocity of blowing air, there are generally two probe sizes, $80 \times 50 \mathrm{~mm}$ and $50 \times 15 \mathrm{~mm}$. The scanning range can be optionally set as to the size of the filter within the size range of the probe scanning mechanism. The latter mode is used for confirming the existence of leakage and its position on the filter judged to have a leakage in the automatic scanning mode. It is possible to choose from two modes: the position control mode, which is operated with the position order; and
Table 1 Specifications

\begin{tabular}{|c|c|c|}
\hline 1 & \multicolumn{2}{|c|}{ Probe Scanning Mechanism } \\
\hline (1) & Size $[\mathrm{mm}]$ & $1,290 \times 750 \times 240^{\mathrm{H}}$ \\
\hline (2) & Weight [kg] & 14.6 \\
\hline (3) & Velocity Control & $\begin{array}{l}\text { Moving at a constant velocity } \\
\text { Auto stopping upon detecting } \\
\text { leakage }\end{array}$ \\
\hline (4) & Position Control & Moving to target position \\
\hline 2 & \multicolumn{2}{|l|}{ Lift Truck } \\
\hline (1) & Size $[\mathrm{mm}]$ & $640 \times 1,100 \times 1700^{\mathrm{H}}$ \\
\hline (2) & Lift Stroke [mm] & 1,890 \\
\hline (3) & Weight $[\mathrm{kg}]$ & 120.7 \\
\hline (4) & Lifting Function & $\begin{array}{l}\text { Manual lifting with handle } \\
\text { Prevention of falling with } \\
\text { ratchet }\end{array}$ \\
\hline (5) & Moving Function & With casters \\
\hline (6) & Positioning & With laser pointer \\
\hline 3 & \multicolumn{2}{|l|}{ Control System } \\
\hline (1) & Apparatus & Light-scattering particle counter \\
\hline (2) & Interface & $\begin{array}{l}\text { Analog input (4-20 mA) } \\
\text { Serial communication (RS232C) }\end{array}$ \\
\hline (3) & Judgment & $\begin{array}{l}\text { Comparison with regulation } \\
\text { value (continuous, total) }\end{array}$ \\
\hline 4 & \multicolumn{2}{|l|}{ Masker } \\
\hline (1) & Size & Depending on filter size \\
\hline (2) & Material & PET and aluminum \\
\hline
\end{tabular}

the velocity control mode, in which the probe moves at a constant velocity. The aspect of the leak test with this machine is shown in Fig. 5. The masker is used in this case. The size of the probe scanning mechanism is designed for testing a $1,200 \times 600 \mathrm{~mm}$ filter, larger filters can be tested by exchanging several parts. The machine has been used to test $1,800 \times 600$ and $1,500 \times 750 \mathrm{~mm}$ filters. Furthermore, the probe scanning mechanism is fixed with the type of buckles so that it is possible to remove it as shown in Fig. 6 and easily pass through doorways. It isalso possible to set the laser pointers on the two corners to adapt the machine position in cases where the masker is not used.

The lift truck has four large casters $200 \mathrm{~mm}$ in diameter and $20 \mathrm{~mm}$ thick, taking into account the unevenness of floors such a grated type. The lift raises the probe scanning mechanism by transmitting the rotation of the handle using the timing belts and pulleys. There is a stopper to halt the handle rotation in the down direction to prevent the scanner from falling down with the weight.

The control system is composed of the programmable logic controller (PLC), the touch panel display and the particle counter. An image of the display in the automatic scanning mode is shown inFig. 7. The scanning of the probe is operated using the touch panel display. The particle counter is connected to the PLC with analog output (4-20 $\mathrm{mA}$ ) and serial communication (RS-232C). The PLC samples 


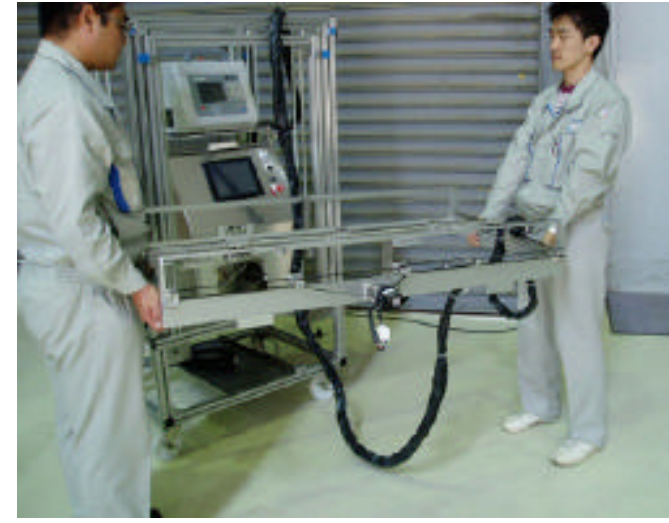

Fig. 6 Removing the probe scanning mechanism

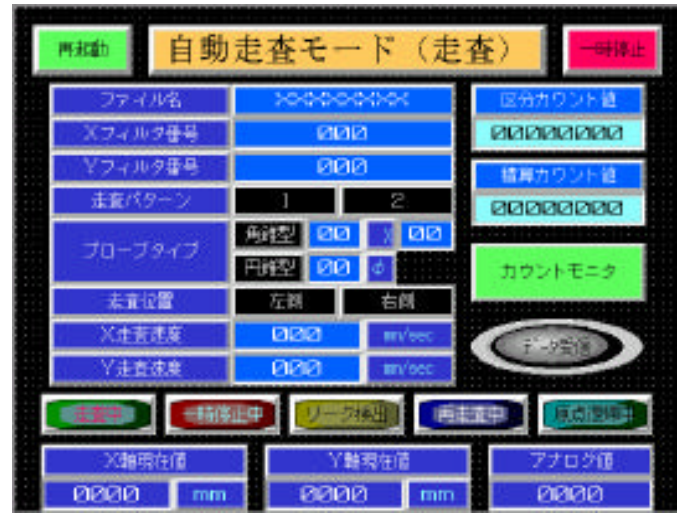

Fig. 7 Example of touch panel display

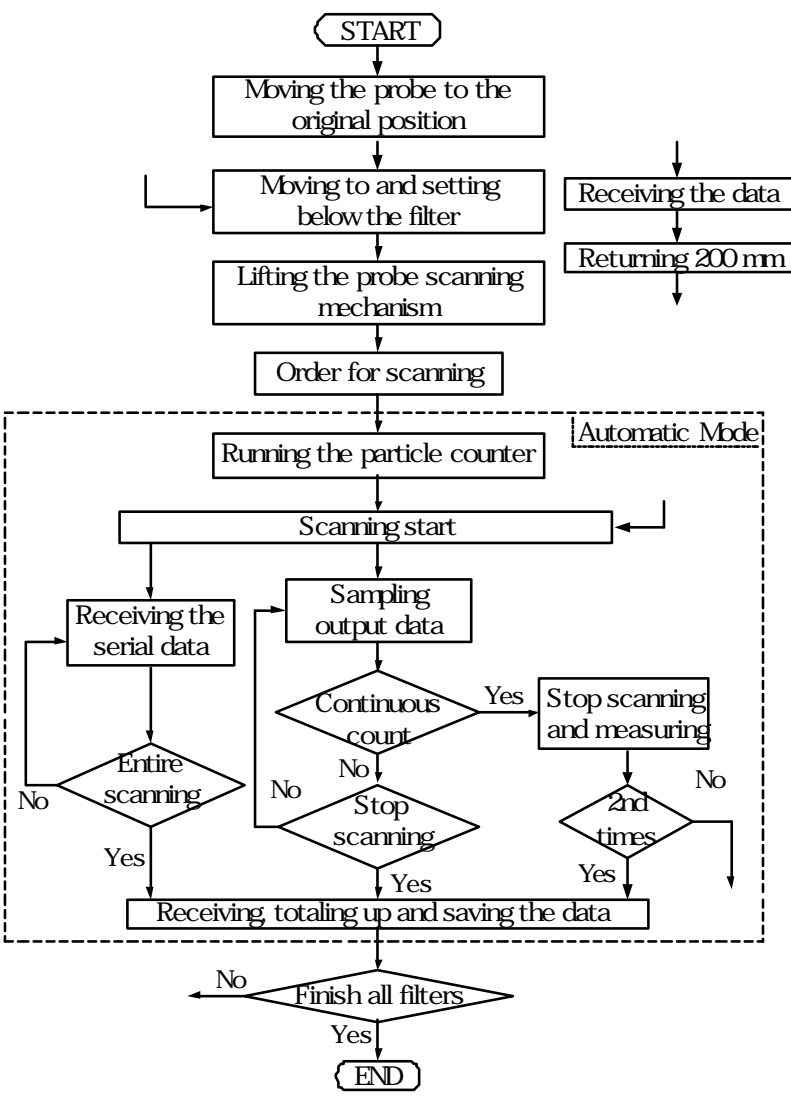

Fig. 8 Flow chart the count data in real time with analog input and the data at constant intervals with serial communication.

\subsection{Test procedure}

The procedure for conducting the leak test using this machine is shown in Fig. 8. First, the operator sets the machine just below the filter, raises the probe scanning mechanism and chooses the automatic scanning mode. The machine scans with the probe and detects any leakage automatically. It also automatically sets the probe to the original position at the time of activation. The data counted by the particle counter is sampled by the PLC with analog input and serial communication. The analog input data is sampled at one-second intervals and the machine automatically attempts rescanning (backing up the probe a particular distance and scanning the same line) if one set of sampling data exceeds the regulation value. On the other hand, the serial communication data is sampled and integrated in the control system for a total count. It is possible to optionally set the following two values for the judging conditions:

(1) If analog input at one sampling exceeds the regulation value and the result of rescanning is the same as the previous one.

(2) If the total count exceeds the regulation value.

The count and the judgment are recorded in the compact flash memory of the PLC in text format.

\section{VERIFICATION OF EFFECTS}

We applied this machine at an actual construction site and compared the results with those using the conventional method.

The specifications of the construction are shown below.

(1) Building use: Semiconductor factory

(2) Floor space: $10,000 \mathrm{~m}^{2}$

(3) Specifications of the clean room

(a) Non-unidirectional airflow with diffuser

(b) Cleanliness: Class 1,000

(c) Filter: ULPA

(d) Size of filter: $1,200 * 600 \mathrm{~mm}$

(e) Number of filters: About 3,500

(f) Height of ceiling: 4,300 mm

\subsection{Method used for the leak test}

As described earlier, this machine is capable of testing filters in accordance with JACA Standard No. 24-1989. However, for the test case, it was necessary to remove the diffusers in order to comply with the standard. The time and cost would increase by removing the diffusers prior to conducting the test and replacing them after; in addition, there would be a risk of breaking the filters. Therefore, we decided to conduct the filter leak test without first removing the diffusers. In this case, it was necessary to decide the probe inlet size, which determines the inlet air velocity, the scanning velocity and the distance between the probe and the filter. Because the former two items, the probe inlet size and scanning velocity, would affect the cost as test time, we 
decided that their values complied with the standard: a size of $12 \mathrm{~cm}^{2}$ (inlet air velocity at $0.4 \mathrm{~m} / \mathrm{sec}$ ) and velocity of 50 $\mathrm{mm} / \mathrm{sec}$. We also conducted a preliminary examinationon the distance between the probe and the filter to confirm that leakage detection would be possible.

The filter leak tester was set below the filter that had a pinhole. Outer airwas supplied from upstream and the tester was operated in the automatic mode. The condition for judging was more than five counts per second with the same results obtained for rescanning after backing up $200 \mathrm{~mm}$ (more than five counts per second).

We tried to test for two cases of distance, $100 \mathrm{~mm}$ and 500 $\mathrm{mm}$. In the results, there were some cases in which leakage could not be detected at the distance of $500 \mathrm{~mm}$, presumably due to difficultydetecting particles continuously because of increased particle diffusion with increased distance. Therefore, we determined the distance of $100 \mathrm{~mm}$ between

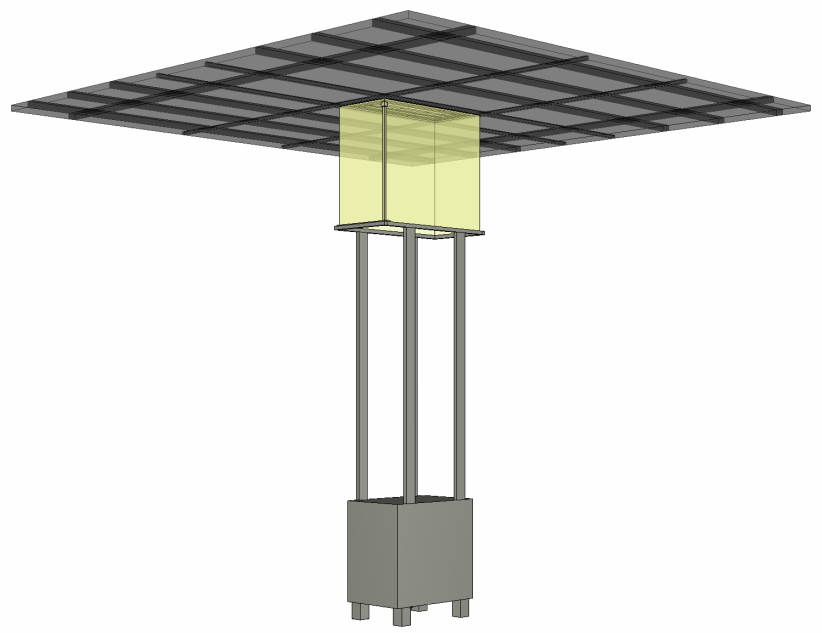

Fig. 9 Simulation model

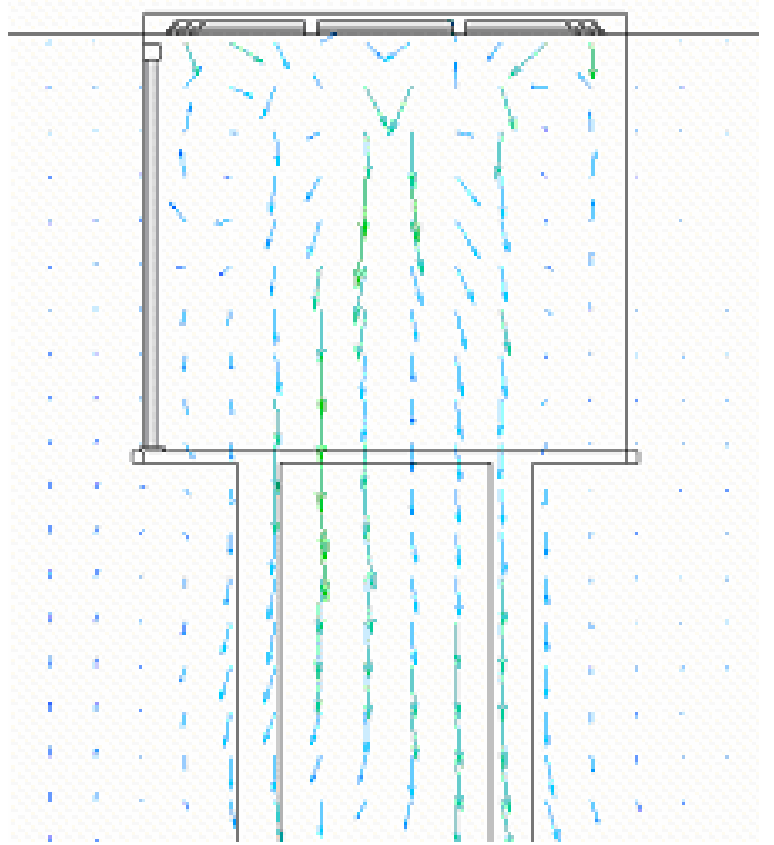

Fig. 10 Longitudinal section the probe and the filter. Additionally, we determined that the conditions for judging were not only the count value at one sampling but also the total count value after scanning. Our objective was to improve the reliability of results in consideration of cases where continuous count was not possible due to the diffusion.

Furthermore, there was a concern that outside air was led into the masker from the bottom because the velocity of the blowing air was accelerated by the diffuser. Therefore, we confirmed this with CFD simulation, using STREAM Ver. 3.12 (Software Cradle Co., Ltd.) with 4,870,000 as the number of divisions forcalculation. The simulation model is shown in Fig. 9 and examples of the longitudinal section andlateral one are shown in Figs. 10 and 11. As seen in these figures, there is no air induced from the bottom.

\subsection{Test results}

Four of the machines were used at the site. The duration of the filter leak test was 15 days. Fig. 12 shows the test results, which lists the number of filters that each machine tested each day, and includes the number of retested filters after exchanging. Itwas found that 139 filters were tested by one machine perday ( 8.5 hours) at the most. The reason why the number of filters at the beginning and end of the term are comparatively small is because the test proceeded as construction progressed.

\subsection{Comparison with conventional method}

We compared the results with those of the conventional method for the same scale of clean room in order to evaluate the effects on time andcost reduction. The results were from a test conducted in a clean room with non-unidirectional airflow so polyethylene sheets were set below all filters before testing.

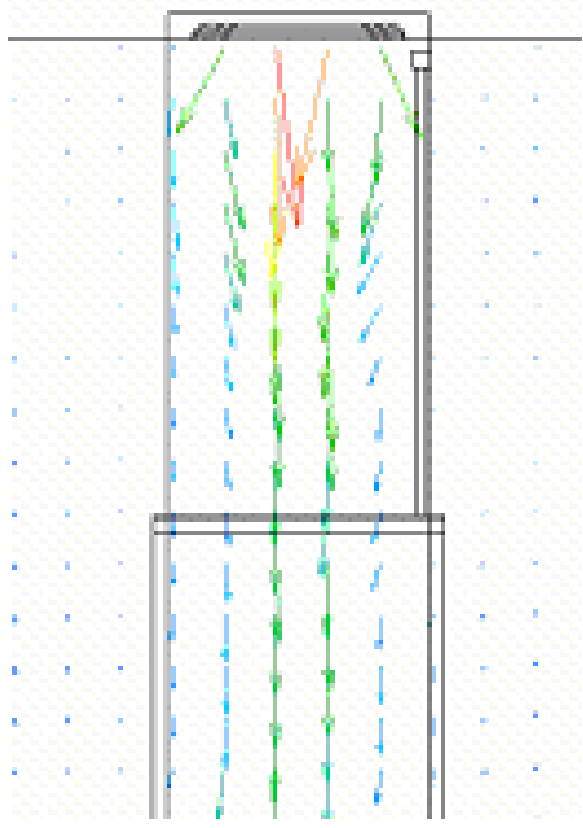

Fig. 11 Lateral section 


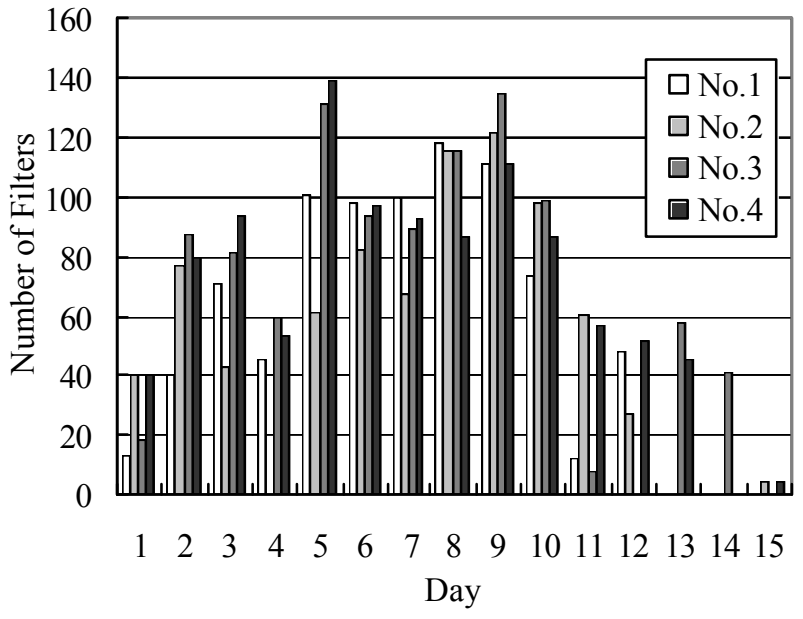

Fig. 12 Test results

It was found that the volume of work using the filter leak tester is about a quarter of that using the conventional method.

It is difficult tomake a precise comparison of the work time because it depends on the progress of the construction, but a general comparison of the results is as follows:

Conventional method: 27 days

Filter leak tester: 15 days

A reduction of 12 days (44\%) was realized. Here, the time for setting the polyethylene sheets in the conventional method was not added to the total time because the sheets can be set along with conducting the leak test.

\section{CONCLUSION}

This report described the specifications of the filter leak tester that we developed for clean rooms, and the effects calculated from the results of application at an actual construction site. We confirmed that it is possible to achieve a significant reduction in time and cost in comparison with the conventional method. However, the machine is still heavy in spite of making it lighter than previously developed automatic robots, and it is still inconvenient to carry it, move it between rooms and get it over bumps. The effects of the machine would be small compared with the trouble of carrying and moving it among certain types of clean rooms, for example, those that are small and have many partitions; therefore, it is necessary tofurther reduce the weight. On the other hand, it is also necessary to cope with increases in ceiling height and filter size in accordance with increased semiconductor and LCD production equipment. Further work is necessary to resolve these conflicting problems.

\section{REFERENCES}

[1] K. Tanaka, et al.: Development of a Construction Robot for Marking on Ceiling Boards, Trans. of the Japan Society of Mechanical Engineers (C), 62-594, pp. 658-664 (1996)

[2] K. Tanaka, et al.: Development of a Construction Robot for Marking on Ceiling Boards, Journal of Robotics and Mechatronics, Vol. 9, No. 4, pp. $293-298$ (1997)

[3] Y. Abe, et al.: Development of Air Conditioning Equipment Inspection Robot for Automatic Measurement of Outlet Air Volume, Journal of the Robotics Society of Japan, Vol. 14, No. 5, pp. 720-732 (1996)

[4] Y. Abe, et al.: Navigation System for Air Conditioning Equipment Inspection Robot, Journal of Robotics and Mechatronics, Vol. 7, No. 5, pp. 354-366 (1995)

[5] T. Nishioka, Research and Development of a Clean Room Test Robot "CRIMRO”. Robot, No. 86, pp. 20-26 (1992)

[6] T. Yoshida, Automating of Inspection with Leak Test Robot, Heating Piping and Air Conditioning, Vol. 27, No. 12, pp. 68-72 (1989) 